Arq. Bras. Med. Vet. Zootec., v.70, n.2, p.429-436, 2018

\title{
Índice de normalidade da distância atlantoaxial dorsal em cães
}

[Normal index of dorsal atlantoaxial distance in dogs]

\author{
E.A. Tudury, A.C. Silva, M.A.S. Lacerda, B.M. Araújo, M.M.A. Amorim, J.E.B. Leite
}

Universidade Federal Rural de Pernambuco - UFRPE - Recife, PE

\begin{abstract}
RESUMO
Foram utilizados 30 cães neurologicamente saudáveis e realizadas radiografias da coluna cervical, sendo mensurada a menor distância atlantoaxial dorsal e outros parâmetros anatômicos vertebrais. Esses dados foram avaliados por meio do coeficiente de correlação de Pearson. Após detecção do melhor parâmetro de correlação, utilizou-se a fórmula de coeficiente de correlação dimensional (CCD). Na segunda etapa desta pesquisa, foram utilizadas imagens radiográficas de 33 cães com subluxação atlantoaxial (SAA) e aplicou-se a fórmula CCD. Todas as imagens atlantoaxiais normais e subluxadas foram distribuídas aleatoriamente e submetidas à análise subjetiva. Observou-se que o parâmetro anatômico de maior correlação foi o comprimento do processo espinhoso do áxis, obtendo-se valor de índice médio de $0,056 \mathrm{e}$ desvio-padrão de 0,019 . O valor de índice médio obtido em cães com SAA foi de 0,287 , e o desviopadrão de 0,123 . A análise subjetiva revelou acerto de $88,89 \%$ a $95,24 \%$, enquanto a avaliação por meio da fórmula CCD demonstrou ser $100 \%$ eficiente no diagnóstico da SAA. Sugeriu-se a utilização da fórmula CCD para obtenção do índice de normalidade da distância atlantoaxial com valor de normalidade médio de 0,056 e limite máximo de 0,098, sendo esse índice eficaz no diagnóstico da subluxação entre o atlas e o áxis.
\end{abstract}

Palavras-chave: atlas, áxis, instabilidade, neurologia, subluxação

\begin{abstract}
Thirty healthy dogs had their cervical spines radiographed, and the dorsal atlantoaxial distance and others vertebral anatomical parameters were measured. These data were assessed using the Pearson correlation coefficient. After detection of the best correlation parameter, we used the dimensional correlation coefficient of formula (CCD). In the second stage of the study, radiographs of the 33 dogs with atlantoaxial subluxation (SAA) were used. In each radiographic image, the CCD formula was applied. All normal and subluxation radiographic images were randomized, and submitted to subjective analysis. It was observed that the anatomical parameter with the highest correlation is the length of the spinous process of the axis, obtaining a mean value of 0.056 index and 0.019 standard deviation. Index mean value obtained in dogs with the disease of 0.287 and standard deviation of 0.123 . The subjective analysis revealed $88.89 \%$ to $95.24 \%$ agreement, while the result obtained by the CCD formula was shown to be $100 \%$ effective in the diagnosis of SAA. We suggest the use of the CCD formula to obtain the index of normality of the atlantoaxial distance with a mean value of 0.056 and maximum of 0.098 , and it is concluded that the index is highly effective in the diagnosis of subluxation between the atlas and the axis.
\end{abstract}

Keywords: atlas, axis, instability, neurology, subluxation

Recebido em 14 de novembro de 2016

Aceito em 20 de abril de 2017

E-mail: eat@dmv.ufrpe.br 


\section{INTRODUÇÃO}

A instabilidade atlantoaxial, também descrita como subluxação atlantoaxial (SAA), é uma alteração existente entre as duas primeiras vértebras cervicais (atlas e áxis), que pode resultar de malformações congênitas, como a ausência ou a hipoplasia do processo odontoide, e/ou traumas (Chrisman et al., 2005; Seim, 2008; Fernández e Bernardini, 2010; Widmer e Thrall, 2013), causando deslocamento dorsal e cranial do áxis, e seu afastamento em relação ao arco dorsal do atlas, com consequente compressão da porção cervical cranial da medula espinal e de suas raízes nervosas, gerando dor e deficiências neurológicas em graus variáveis, desde ligeira ataxia e deficiências proprioceptivas até tetraparesia grave (Seim, 2008; Fernández e Bernardini, 2010; Lorenz et al., 2011).

Essa afecção ocorre mais frequentemente em cães de raças toy ou miniatura, tais como Poodle, Pinscher e Yorkshire Terrier (Beaver et al., 2000; Beckmann et al., 2010). A maioria dos cães que apresentam sinais por essa afecção tem menos de um ano de idade (Lorenz e Kornegay, 2006; Seim, 2008, Fernández e Bernardini, 2010; Lorenz et al., 2011). Os cães com sinais clínicos em uma idade mais avançada, em geral, têm a anormalidade desde o nascimento, mas um pequeno trauma pode gerar maior instabilidade com compressão significativa da medula espinhal cervical e de suas raízes nervosas (Lorigados et al., 2004; Seim, 2008).

O diagnóstico da SAA é baseado geralmente nos sinais clínicos, sendo confirmado com base nos estudos de imagens, como os achados radiográficos da coluna vertebral cervical em projeção lateral (Lorigados et al. 2004; Widmer e Thrall, 2013), de preferência com o animal acordado, pois uma manipulação excessiva do pescoço em estado de relaxamento muscular pode agravar o quadro clínico (Fernández e Bernardini, 2010; Lorenz et al., 2011). Radiograficamente, em projeção lateral, observase o aumento na distância entre o arco dorsal do atlas e o processo espinhoso do áxis (Kealy e Mcallister, 2005; Seim 2008; Sturges, 2009; Fernández e Bernardini, 2010; Widmer e Thrall, 2013), onde um espaço de mais de 4 a $5 \mathrm{~mm}$ entre a lâmina dorsal do atlas e o processo espinhoso do áxis geralmente permite estabelecer o diagnóstico em um cão de raça pequena (Seim,
2008); o deslocamento dorsal do processo odontoide e/ou corpo do áxis em direção ao canal vertebral também pode(m) ser observado(s), com consequente alteração da relação linear normal entre as lâminas dorsais do atlas e do áxis, formando uma relação angular (Widmer e Thrall, 2013).

McLear e Saunders (2000), em estudo sobre mobilidade da articulação atlantoaxial em cães, determinaram a amplitude de movimentação normal dessa articulação utilizando imagens radiográficas da região cervical cranial em projeção lateral em extensão e flexão, e concluíram que a angulação entre o atlas e o áxis não deve ser inferior a 162 graus em cães normais.

As projeções ventrodorsais com boca aberta e laterais oblíquas evidenciam melhor a ausência ou a fratura do processo odontoide (Seim, 2008; Sturges, 2009; Fernández e Bernardini, 2010). Tomografia e ressonância magnética podem ser utilizadas para definir melhor a anatomia do processo odontoide, mas geralmente não são necessárias para o diagnóstico (Seim, 2008). Sturges (2009) e Middleton et al. (2012) ressaltam a importância da ressonância magnética na visualização de anormalidades referentes às estruturas ligamentares da região occipitoatlantoaxial e na identificação de doenças subjacentes que podem influenciar no prognóstico (Sturges, 2009).

Vários estudos na área de diagnóstico por imagem em medicina veterinária foram desenvolvidos utilizando índices de correlação de medidas entre estruturas anatômicas, a fim de se proporcionar uma avaliação quantitativa, eliminar a subjetividade e anular a interferência do peso e do tamanho do animal. Dentre tais estudos, pode-se citar o método VHS (vertebral heart size) para detecção do aumento generalizado do coração (Buchanan e Bücheler, 1995); a relação entre o diâmetro do cólon e o comprimento da quinta vértebra lombar na determinação de constipação e megacólon em gatos (Trevail et al., 2011); a relação entre o comprimento hepático e a $11^{\mathrm{a}}$ vértebra torácica na detecção de hepatomegalia (Lopes et al., 2011); o tamanho dos rins com referência à segunda vértebra lombar (Farrow, 2006); o índice de distração para detecção de displasia coxofemoral em cães jovens, obtido pelo método 
PennHip (Smith et al., 1990); o índice de subluxação dorsolateral da articulação coxofemoral que avalia a subluxação passiva da cabeça femoral, útil no diagnóstico de displasia coxofemoral (Farese et al., 1998); a relação entre o edema da medula espinal e o comprimento da segunda vértebra lombar, na determinação do prognóstico de cães com doença do disco intervertebral (Duval et al.; 1996); entre outros.

Objetivou-se com este trabalho determinar um índice de normalidade da distância atlantoaxial dorsal de cães, por meio de uma fórmula de coeficente de correlação dimensional, tendo em vista a ausência na literatura veterinária de um parâmetro exato envolvendo a distância atlantoaxial para o diagnóstico da subluxação entre o atlas e o áxis, bem como verificar a eficácia desse índice no diagnóstico da subluxação atlantoaxial em cães.

\section{MATERIAL E MÉTODOS}

Após aprovação do projeto pela Comissão de Ética para Uso de Animais (Ceua) da UFRPE, com devida liberação de licença sob o número 017/2012, foram utilizados 30 animais da espécie canina escolhidos aleatoriamente, sendo 10 da raça Miniatura Pinscher, 10 Yorkshires Terrier, e

a)

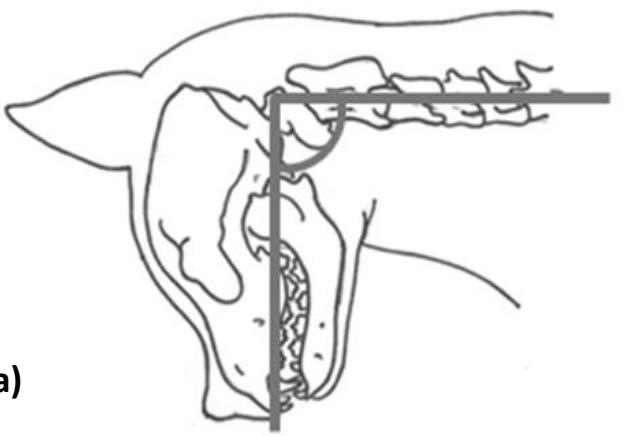

10 Poodles, sem distinção de sexo, com idade entre três meses e 14 anos, com peso igual ou inferior a $6 \mathrm{~kg}$, sem comprometimento neurológico ou espinhal, encaminhados ao Setor de Radiologia do Hospital Veterinário da Universidade Federal Rural de Pernambuco (UFRPE) por motivos não relacionados a este estudo.

A coluna cervical de cada animal foi radiografada nas projeções lateral, com 90 e 45 graus de flexão (Fig. 1), e ventrodorsal, sendo mensurada a menor distância existente entre o arco dorsal do atlas e a borda cranioventral do processo espinhoso do áxis (MDAA), o comprimento do processo espinhoso do áxis (CPEA), o comprimento do arco dorsal do atlas (CADA), a altura da porção caudal do corpo vertebral do áxis (APCCVA), a altura do canal vertebral caudal do áxis (ACVA) e o comprimento dorsal do corpo da terceira vértebra cervical (CC3) (obtidos nas projeções laterais); a largura da porção cranial do corpo do áxis (LCA) e a largura do atlas (LA) correspondente às extremidades das asas do atlas (obtidas na projeção ventrodorsal). Para obtenção das radiografias, foi utilizada contenção física, dispensando-se a realização de anestesia.

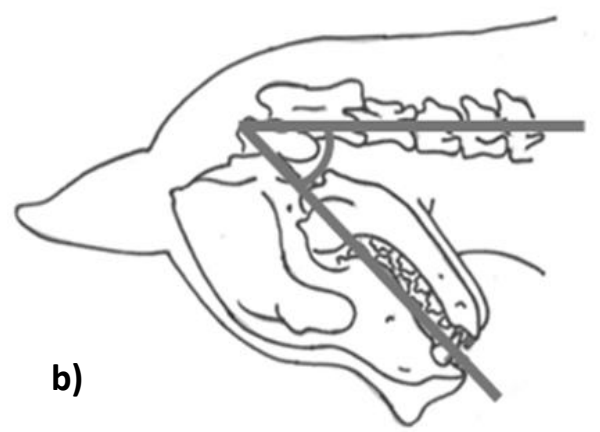

Figura 1. Desenho esquemático do posicionamento dos animais para obtenção das radiografias da coluna cervical nas projeções laterais, com 90 graus de flexão (a) e 45 graus de flexão (b) do pescoço.

Após a obtenção dos dados, estes foram avaliados por meio do coeficiente de correlação (r) de Pearson, a fim de se identificar o parâmetro anatômico de melhor correlação com a distância atlantoaxial dorsal e com mensuração mais prática e confiável. Após identificação desse melhor parâmetro de correlação, utilizouse a fórmula abaixo (coeficiente de correlação dimensional - CCD), com o intuito de se alcançar um índice de normalidade aplicável a vários cães toy, independentemente da raça, do tamanho e do peso.

$\mathrm{CCD}=\frac{\text { MDAA }}{\mathrm{CPEA}}$

em que:

$\mathrm{CCD}=$ coeficiente de correlação dimensional

MDAA = menor distância entre o arco dorsal do atlas e o processo espinhoso do áxis em 90 graus de flexão 


\section{Tudury et al.}

$\mathrm{CPEA}=$ comprimento do processo espinhoso do áxis.

Na segunda etapa deste estudo, foram utilizadas imagens radiográficas da coluna vertebral cervical em projeção lateral, com 90 graus de flexão ou angulações superiores (mais próximas da extensão do pescoço), de 33 cães com SAA, sem distinção de raça, sexo, peso e idade, obtidas por meio de arquivos pessoais próprios e de vários neurologistas veterinários do Brasil.

Em cada imagem radiográfica, foi mensurada a menor distância existente entre o arco dorsal do atlas e a borda cranioventral do processo espinhoso do áxis (MDAA), e o comprimento do processo espinhoso do áxis (CPEA) (Fig. 2). Após a obtenção dos dados, estes foram aplicados à fórmula CCD.

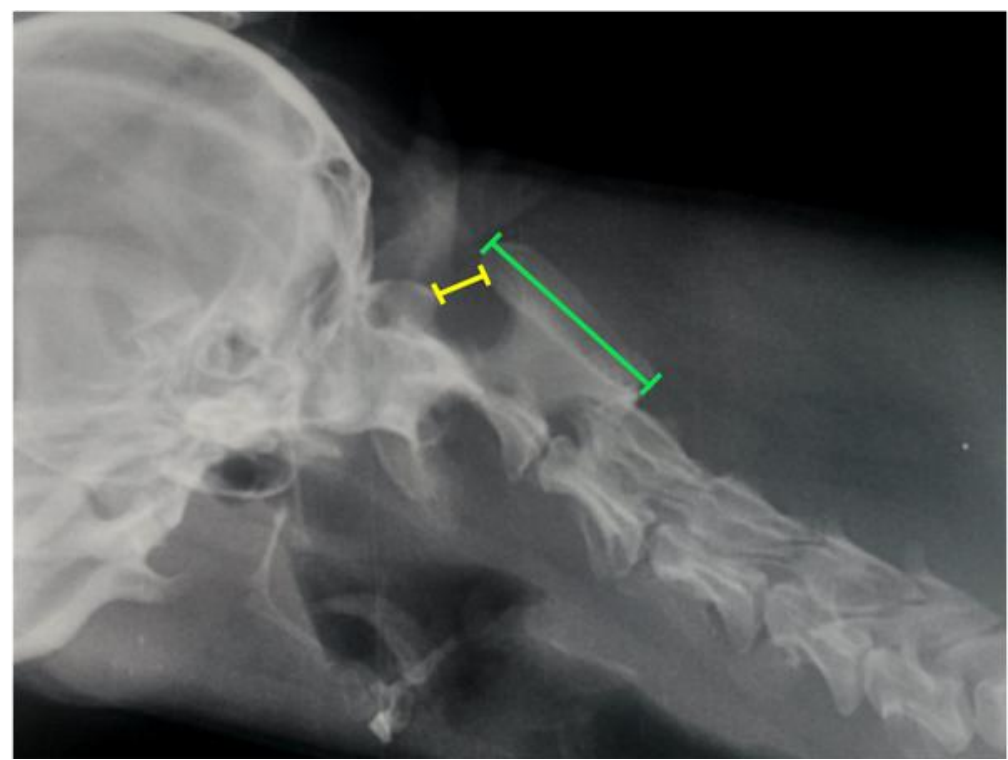

Figura 2. Imagem radiográfica ilustrativa das distâncias mensuradas em projeção lateral com aproximadamente 90 graus de flexão do pescoço, em um cão da raça Yorkshire com subluxação atlantoaxial.

( amarelo $)=$ Menor distância entre o arco dorsal do atlas e a borda cranioventral do processo espinhoso do áxis (MDAA).

- $($ verde $)=$ Comprimento do processo espinhoso do áxis (CPEA).

As 33 imagens radiográficas de SAA juntamente com as 30 imagens radiográficas da articulação atlantoaxial normais foram distribuídas aleatoriamente e submetidas à análise subjetiva por três profissionais experientes em neuroimagem, com o intuito de se comparar a eficiência entre a avaliação subjetiva e a avaliação obtida por meio da fórmula CCD.

Todas as imagens radiográficas (com e sem SAA) foram submetidas à avaliação da relação angular entre o atlas e o áxis proposta por McLear e Saunders (2000).

Os dados foram organizados e analisados por meio de tabelas, gráficos, medidas de tendência central (média e mediana), medidas de dispersão (desvio-padrão), coeficiente de correlação (r) mediante o uso da correlação de Pearson, e testes de hipóteses: teste paramétrico $\mathrm{t}$ pareado para avaliação dos resultados obtidos da MDAA com flexão de 90 e 45 graus; teste ANOVA para avaliação dos índices de normalidade obtidos nos três grupos de raças distintas; e teste paramétrico t de Student para avaliação das diferenças entre os resultados de índices obtidos nos cães afetados em comparação com os índices de normalidade obtidos nos cães normais de raças toy; comparação dos índices obtidos em animais jovens sadios (com idade menor ou igual a 12 meses) versus adultos sadios (com idade superior a 12 meses); jovens afetados versus adultos afetados; jovens afetados versus jovens sadios; e adultos afetados versus adultos sadios, sendo 
utilizado o nível de significância de 5\%. O software utilizado foi o GraphPad InStat, versão 3.05.

Licença emitida pela Comissão de Ética para Uso de Animais (Ceua) da UFRPE: 017/2012.

\section{RESULTADOS E DISCUSSÃO}

As raças Miniatura Pinscher, Yorkshire Terrier e Poodle foram escolhidas para determinação de índice de normalidade, pelo fato de a instabilidade atlantoaxial ocorrer mais frequentemente em cães de raças toy ou miniatura. Dentre os animais afetados, as raças mais acometidas pela SAA, segundo dados obtidos neste estudo, foram Yorkshire Terrier $(30,30 \%)$, Poodle $(21,21 \%)$, cães sem raça definida $(12,12 \%)$, Miniatura Pinscher $(9,09 \%)$ e Lhasa Apso (9,09\%), entretanto a afecção também foi verificada em cães de outras raças, como Maltês $(6,06 \%)$, Chihuahua $(6,06 \%)$ e Lulu da Pomerânia $(3,03 \%)$, e ainda em um cão da raça Schnauzer $(3,03 \%)$, o que corrobora Beckmann et al. (2010) ao afirmarem que a SAA é mais frequentemente observada em cães de raças toy ou miniatura, contudo outras raças podem ser acometidas.

A idade dos animais afetados variou de três a 108 meses, mas 72,72\% dos animais acometidos tinham idade igual ou inferior a 12 meses, o que mostra que a afecção em questão é mais frequente em animais jovens, sobretudo durante o primeiro ano de vida, como citam Lorenz e Kornegay (2006), Seim (2008), e Beckmann et al. (2010), podendo ocorrer mais tardiamente em animais que geralmente têm a instabilidade desde o nascimento, mas um pequeno trauma pode causar a ruptura dos ligamentos com compressão significativa da medula espinal e raízes nervosas, desencadeando os sinais clínicos (Seim, 2008).

O intuito do presente estudo foi auxiliar no diagnóstico da instabilidade atlantoaxial, tendo em vista a subjetividade da avaliação radiográfica, corroborando Platt e Costa (2012) ao afirmarem que, sem uma medição objetiva, alguns cães podem ser incorretamente diagnosticados.

No diagnóstico da SAA, o principal achado é o aumento na distância entre o arco dorsal do atlas e o processo espinhoso do áxis (Kealy e
Mcallister, 2005; Fernández e Bernardini, 2010). Seim (2008) descreve que um espaço de mais de 4 a $5 \mathrm{~mm}$ entre a lâmina de $\mathrm{C} 1$ e a espinha dorsal de C2 geralmente permite estabelecer o diagnóstico em um cão de raça pequena. Apesar da descrição de valores numéricos por Seim (2008), estes são imprecisos, diante da grande variabilidade de tamanho dos cães, mantendo-se, assim, a subjetividade.

Outro parâmetro observado comumente de forma subjetiva no diagnóstico da SAA em radiografias em projeção lateral é o deslocamento dorsal do processo odontoide e do corpo do áxis em direção ao canal vertebral, com consequente formação de relação angular dorsal entre a lâmina dorsal do atlas e a lâmina dorsal do áxis (Widmer e Thrall, 2013). Entretanto, neste estudo observou-se que, em sete casos $(21,21 \%)$, não ocorreu a alteração da relação linear normal, logo, não houve a citada relação angular como descrita por Widmer e Thrall (2013). Dessa forma, esse sinal radiográfico não é $100 \%$ eficiente no diagnóstico da SAA, já que esse achado não se fez presente em todos os casos.

O parâmetro angular proposto por McLear e Saunders (2000) aplicado nas imagens radiográficas normais com angulação cervical de 90 graus resultou em valor médio de 181,46 graus, desvio-padrão de 6,383 e valores mínimo e máximo de 172 e 197 graus, respectivamente; enquanto nas imagens radiográficas com angulação forçada de 45 graus, o valor médio foi de 175,40 , o desvio-padrão de 5,858 e os valores mínimo e máximo de 165 e 189 , respectivamente. Logo, os resultados obtidos neste estudo estão de acordo com o estudo de mobilidade atlantoaxial realizado por McLear e Saunders (2000), que determina que a angulação entre o atlas e o áxis não deve ser inferior a 162 graus em cães normais. Todavia, ao se aplicar o referido parâmetro nas imagens radiográficas de cães com sinais clínicos e radiográficos de SAA, o valor médio foi de 140,45 graus, o desviopadrão de 20,878 e os valores mínimo e máximo de 92 e 176 graus, respectivamente. Três $(9,09 \%)$ animais, radiografados com o pescoço em 90 graus de flexão, apresentaram angulação superior a 162 graus, valor esse preestabelecido como limite inferior de normalidade, demonstrando, assim, que esse método não é $100 \%$ eficiente. 
Quanto à realização das radiografias sem a utilização de anestesia, Lorenz e Kornegay (2006) e Fernández e Bernardini (2010) recomendam que, em animais com tal afecção, essas sejam realizadas com o animal acordado, pois cães anestesiados não mantêm a tensão muscular cervical, o que aumenta a possibilidade de flexão do pescoço e grave compressão da medula espinhal, logo, se na situação patológica não se recomenda anestesia, no presente estudo de normalidade foi seguida a mesma orientação, evitando que o relaxamento da musculatura cervical resultasse em valores de mensuração diferentes aos de cães acordados com a afecção.

As mensurações dos parâmetros anatômicos do atlas, do áxis e da terceira vértebra cervical foram avaliadas por meio da correlação de Pearson, em que o parâmetro anatômico de maior correlação com a distância atlantoaxial dorsal foi o comprimento do processo espinhoso do áxis $(\mathrm{r}=0,6005)$.

A fórmula utilizada $(\mathrm{CCD}=\mathrm{MDAA} \div \mathrm{CPEA})$ foi desenvolvida com o propósito de determinar o índice de normalidade da distância atlantoaxial por meio de um coeficiente de correlação, de forma que variáveis como peso, tamanho e raça fossem anuladas, assim como observado em outros estudos envolvendo correlações de estruturas anatômicas. Diante da utilização do comprimento do processo espinhoso na fórmula CCD, obteve-se valor de índice de normalidade médio de 0,056 (oscilando de 0,022 a 0,098) e desvio-padrão de 0,019 nas radiografias em projeção lateral com 90 graus de flexão do pescoço.

Os valores de índices obtidos por meio da fórmula CCD nos cães com a afecção variaram de 0,120 a 0,627 , com valor médio de 0,287 e desvio-padrão de 0,123 , logo, observou-se que todos os valores foram superiores aos obtidos em cães normais.

$\mathrm{Na}$ análise estatística para avaliação das diferenças de índices obtidos neste estudo em comparação com os índices de normalidade, foi obtido valor de $\mathrm{P}<0,0001$, o que assinala a eficácia diagnóstica do índice de normalidade da distância atlantoaxial dorsal em cães, independentemente do grau de flexão do pescoço, considerando-se que foram utilizadas imagens radiográficas cervicais laterais com angulações de aproximadamente 90 graus de flexão e com angulações superiores (mais próximas da extensão do pescoço).

Esta ferramenta pode ser empregada rotineiramente diante da suspeita clínica da SAA, tendo em vista que anula a subjetividade da avaliação radiográfica e constitui um método rápido, objetivo, prático, de fácil reprodução, independentemente do grau de experiência do profissional e da variabilidade de tamanho dos animais.

A projeção lateral com 90 graus de flexão foi escolhida para obtenção dos resultados, uma vez que, diante de uma instabilidade atlantoaxial, a flexão excessiva (45 graus) poderia resultar em maior compressão medular, exacerbando os déficits neurológicos, com possibilidade de paralisia respiratória e óbito (Seim, 2008).

$\mathrm{Na}$ análise estatística para avaliação dos resultados obtidos entre as flexões de 90 e 45 graus, foi obtido valor de $\mathrm{P}<0,0001$, considerado significativo, o que reforça o cuidado quanto à manipulação com flexão excessiva e a não realização de sedação em pacientes que apresentem a instabilidade atlantoaxial.

A idade dos animais utilizados neste estudo de normalidade variou de quatro meses a 14 anos, entretanto, estatisticamente, os índices obtidos em animais jovens $(\leq 12$ meses $)$ não apresentaram diferença $(\mathrm{P}=0,2720)$ quando comparados com animais adultos (>12 meses), o que demonstra que o ligamento atlantoaxial dorsal não se apresenta mais frouxo em filhotes, como ocorre, por exemplo, com ligamento cruzado cranial do joelho de cães jovens, que pode permitir um deslocamento cranial da tíbia de até $4 \mathrm{~mm}$ durante o movimento de gaveta, em articulações normais (Moore e Red, 1996).

Os resultados da avaliação dos índices obtidos nas três raças não diferiram significativamente $(\mathrm{P}=0,7189)$, comprovando que não há necessidade de determinação de um índice de normalidade atlantoaxial para cada uma das raças estudadas.

A análise subjetiva revelou acerto de $88,89 \%$ a $95,24 \%$, enquanto a avaliação obtida por meio da fórmula CCD demonstrou ser $100 \%$ eficiente no diagnóstico da SAA. Vale a pena ressaltar que, 
dentre as imagens que apresentavam SAA, cinco $(7,94 \%)$ foram consideradas inconclusivas pelos avaliadores, por não apresentarem subluxação evidente, apesar de os cães apresentarem sinais clínicos característicos, assim como também foi observado por Lorigados et al. (2004), necessitando da realização de outras projeções para conclusão diagnóstica, logo, ao serem submetidas ao índice, ocorreu a confirmação da suspeita clínica de SAA. Dessa forma, com a utilização do índice de normalidade, a dúvida pôde ser extinta, proporcionando grande auxilio no diagnóstico e poupando a realização de outras projeções capazes de pôr em risco a vida do paciente.

Estatisticamente, os índices obtidos em animais jovens afetados $(\leq 12$ meses) não apresentaram diferença significativa $(\mathrm{P}=0,4530)$ quando comparados aos dos animais adultos afetados (>12 meses), o que demonstra que o índice tem ótima aplicabilidade independentemente da idade, assim como observado no estudo anterior com animais normais; enquanto os índices obtidos em animais jovens afetados apresentaram diferença significativa $(\mathrm{P}<0,0001)$ quando comparados aos dos animais jovens sadios, assim como também observado entre animais adultos afetados e adultos sadios, indicando a confiabilidade do índice para as diferentes situações (normais/afetados).

Lamb e Nelson (2014) colocam em dúvida a validade das medições radiológicas de vários estudos, devido a alguns fatores, como a variabilidade fenotípica de tamanho de estruturas dos cães; a ausência de comparação entre as avaliações subjetivas e os métodos com medições, considerando-se que, muitas vezes, os avaliadores obtêm a mesma eficiência que as medições; e a dificuldade no acerto da determinação das medidas utilizadas para realização dos métodos, fatores esses não ocorridos ou eliminados neste trabalho, tendo em vista que, com o índice da distância atlantoaxial dorsal, a variabilidade fenotípica foi anulada, o método utilizado foi comparado com a avaliação subjetiva (e o índice teve maior eficácia que os avaliadores), e as duas medidas (distância atlantoaxial dorsal e comprimento do processo espinhoso do áxis) não têm complexidade nem dificuldade de serem mensuradas.

\section{CONCLUSÃO}

Com base nos resultados obtidos neste estudo, sugere-se a utilização da fórmula CCD para obtenção do índice de normalidade da distância atlantoaxial com valor de normalidade médio de 0,056 e limite máximo de 0,098, sendo esse índice eficaz no diagnóstico da subluxação entre o atlas e o áxis, independentemente do tamanho e da idade dos cães.

\section{AGRADECIMENTOS}

Aos professores André Luis Selmi (Anhembi Morumbi - SP), Alexandre Mazzanti (UFSM RS), Leonardo Augusto Lopes Muzzi (UFLA MG), Hélio José Santos Bagetti Filho (Unifeso RJ), Vitor Márcio Ribeiro (PUC - MG) e Mônica Vicky Bahr Arias (UEL - Paraná), pela disponibilidade e concessão de imagens radiográficas para realização deste estudo. Ao $\mathrm{CNPq}$, pela bolsa oferecida, que contribuiu para a realização desta pesquisa.

\section{REFERÊNCIAS}

BEAVER, D.P.; ELLISON, G.W.; LEWIS, D.D. et al. Risk factors affecting the outcome of surgery for atlantoaxial subluxation in dogs: 46 cases (1978-1998). J. Am. Vet. Med. Assoc. v.216, p.1104-1109, 2000.

BECKMANN, D.V.; MAZZANTI, A.; SANTINI, G. et al. Subluxação atlantoaxial em 14 cães (2003-2008). Pesqui. Vet. Bras. v.30, p.172-176, 2010.

BUCHANAN, J.W.; BÜCHELER, J. Vertebral scale system to measure canine heart size in radiographs. J. Am. Vet. Med. Assoc., v.206, p.194-199, 1995.

CHRISMAN, C.; MARIANI, C.; PLATT, S. et al. Quadriparesia, quadriplegia, hemiparesia, hemiplegia agudas. In: Neurologia para o clínico de pequenos animais. São Paulo: Roca, 2005. cap.9, p.203-225.

DUVAL, J.; DEWER, C.; ROBERTS, R.; ARON, D. Spinal cord swelling as a myelographic indicator of prognosis: a retrospective study in dogs with intervertebral disc disease and loss of deep pain perception. Vet. Surg. v.25, p.6-12, 1996. 
FARESE, J.P.; TODHUNTER, R.J.; LUST, G. et al. Dorsolateral subluxation of hip joints in dogs measured in a weight-bearing position with radiography and computed tomography. Vet. Surg. v.27, p.393-405, 1998.

FARROW, C.S. Doenças dos rins, ureteres, bexiga urinária e uretra. In: Veterinária diagnóstico por imagem do cão e do gato. São Paulo: Roca, 2006. cap.70. p.677-712.

FERNÁNDEZ, V.L.; BERNARDINI, M. Enfermidades da medula com afecção neurológica secundária. In:

Neurologia em cães e gatos. São Paulo: MedVet, 2010. cap.15. p.319-366.

KEALY, J.K.; MCALLISTER, H. Radiologia $e$ ultra-sonografia do cão e do gato. 3.ed. São Paulo: Manole, 2005. p.339-411.

LAMB, C.R.; NELSON, J.R. Diagnostic accuracy of tests based on radiologic measurements of dogs and cats: a systematic review. Vet. Radiol. Ultrasound, v.56, p.231244, 2015.

LOPES, B.F.; TAFFAREL, M.O.; FEITOSA, M.L. et al. Radiografia quantitativa hepática de cães tranquilizados com acepromazina. Cienc. Rural, v.41, p.137-142, 2011.

LORENZ, M.D.; COATES, J.R.; KENT, M. Tetraparesis, hemiparesis, and ataxia. In: Handbook of veterinary neurology. 5.ed.

St. Louis: Elsevier Saunders, 2011. chap.7, p.162-249.

LORENZ, M.D.; KORNEGAY, J.N. Tetraparesia, hemiparesia e ataxia. In: $\overline{\text { Manole, }}$. 2006. cap.7, p.175-218.

LORIGADOS, C.A.B.; STERMAN, F.A.; PINTO, A.C.B.F. Clinic-radiographic study of congenital atlantoaxial subluxation in dogs. Braz. J. Vet. Res. Anim. Sci. v.41, p.368-374, 2004.
McLEAR, R.C.; SAUNDERS, H.M. Atlantoaxial mobility in the dog. Vet. Radiol. Ultrasound, v.41, p.558, 2000.

MIDDLETON, G.; HILLMANN, D.J.; TRICHEL, J. et al. Magnetic resonance imaging of the ligamentous structures of the occipitoatlantoaxial region in the dog. Vet. Radiol. Ultrasound, v.53, p.545-551, 2012.

MOORE, K.W.; READ, R.A. Rupture of cranial cruciate ligament in dogs. Part II. Diagnosis and management. Comp. Cont. Educ. Pract. Vet. v.18, p.381-391, 1996.

PLATT, S.R.; COSTA, R.C. Cervical spine. In: TOBIAS, K.M.; JOHNSTON, S.A. Veterinary surgery small animal. St. Louis: Elsevier Saunders, 2012. cap.31, p.410-448.

SEIM, H.B. Cirurgia da coluna cervical. Instabilidade atlantoaxial. In: FOSSUM, T.W. Cirurgia de pequenos animais. 3.ed. Rio de Janeiro: Elsevier, 2008. cap.38, p.1441-1446.

SMITH, G.K.; BIERY, D.N.; GREGOR, T.P. News concepts of coxofemoral joint stability and the clinical stress-radiographic method for quantification hip joint laxity in the dog. J. Am. Vet. Med. Assoc., v.196, p.59-70, 1990.

STURGES, B.K. Diagnosis and treatment of atlantoaxial subluxation. In: BONAGURA, J.D.; TWEDT, D.C. Kirk's current veterinary therapy $X I V$. St. Louis: Saunders Elsevier, 2009. chap.236, p.1083-1087.

TREVAIL, T.; GUNN-MOORE, D.; CARRERA, I. et al. Radiographic diameter of the colon in normal and constipated cats and in cats with megacolon. Vet. Radiol. Ultrasound, v.52, p.516-520, 2011.

WIDMER, W.R.; THRALL, D.E. The canine and feline vertebrae. In: THRALL, D.E. Textbook of veterinary diagnostic radiology. 6.ed. St. Louis: Elsevier Saunders, 2013. chap.11, p.172-193. 